University of Wollongong

Research Online

Australian Institute for Innovative Materials -

Papers

Australian Institute for Innovative Materials

$1-1-2008$

High field performance of nano-diamond doped MgB2 superconductor

Arpita Vajpayee

National Physical Laboratory, India

V P. Awana

National Physical Laboratory, India

H Kishan

National Physical Laboratory, India

Anant Narlikar

UGC-DAE Consortium for Scientific Research, India

G L. Bhalla

Delhi University, India

See next page for additional authors

Follow this and additional works at: https://ro.uow.edu.au/aiimpapers

Part of the Engineering Commons, and the Physical Sciences and Mathematics Commons

Research Online is the open access institutional repository for the University of Wollongong. For further information contact the UOW Library: research-pubs@uow.edu.au 


\title{
High field performance of nano-diamond doped MgB2 superconductor
}

\author{
Abstract \\ The results from magnetotransport and magnetization of nanodiamond doped $\mathrm{MgB}_{2}-\mathrm{nD}_{\mathrm{x}}$ are reported. \\ Superconducting transition temperature $\left(T_{C}\right)$ is not affected significantly by $x$ up to $x=0.05$ and latter \\ decreases slightly for higher $x>0.05$. $R(T)$ vs $H$ measurements show higher $T_{C}$ values under same applied \\ magnetic fields for the nanodiamond added samples, resulting in higher estimated $\mathrm{Hc}_{2}$ values. From the \\ magnetization measurements, it was found that irreversibility field value $\mathrm{H}_{\text {irr }}$ for the pristine sample is 7.5 \\ $\mathrm{T}$ at $4 \mathrm{~K}$ and the same is increased to $13.5 \mathrm{~T}$ for $3 \mathrm{wt} \% \mathrm{nD}$ added sample at the same temperature. The \\ $\mathrm{J}_{\mathrm{C}}(\mathrm{H})$ plots at all temperatures show that $\mathrm{J}_{\mathrm{C}}$ value is lowest at all applied fields for pristine $\mathrm{MgB}_{2}$ and the \\ sample doped with $3 \mathrm{wt} \% \mathrm{nD}$ gives the best $\mathrm{J}_{\mathrm{C}}$ values at all fields. These results are discussed in terms of \\ extrinsic pinning due to dispersed $\mathrm{n}$-diamond in the host $\mathrm{MgB}_{2}$ matrix along with the intrinsic pinning due \\ to possible substitution of $\mathrm{C}$ at boron site and increased interband scattering for highly doped samples \\ resulting in extraordinary performance of the doped system.
}

\section{Keywords}

High, field, performance, nano, diamond, doped, MgB2, superconductor

Disciplines

Engineering | Physical Sciences and Mathematics

\section{Publication Details}

Vajpayee, A, Awana, VP, Kishan, H, Narlikar, A, Bhalla, GL \& Wang, P (2008), High field performance of nano-diamond doped MgB2 superconductor, In 52nd Annual Conference on Magnetism and Magnetic Materials (MMM), NOV 05-09, 2007, Tampa, FL, USA, Journal of Applied Physics, 103(7), pp. 1-12.

\section{Authors}

Arpita Vajpayee, V P. Awana, H Kishan, Anant Narlikar, G L. Bhalla, and Xiaolin Wang 


\title{
High field performance of nanodiamond doped $\mathrm{MgB}_{2}$ superconductor
}

\author{
Arpita Vajpayee, ${ }^{\text {a) }}$ V. P. S. Awana, and H. Kishan \\ National Physical Laboratory, Krishnan Marg, New Delhi 110012, India
}

\author{
A. V. Narlikar \\ UGC-DAE Consortium for Scientific Research, University Campus, Khandwa Road, Indore 452017, India
}

G. L. Bhalla

Department of Physics and Astrophysics, Delhi University, New Delhi 110007, India

\author{
X. L. Wang \\ ISEM, University of Wollongong, New South Wales 2522, Australia
}

(Presented on 9 November 2007; received 4 September 2007; accepted 22 October 2007; published online 6 February 2008)

\begin{abstract}
The results from magnetotransport and magnetization of nanodiamond doped $\mathrm{MgB}_{2}-n \mathrm{D}_{x}$ are reported. Superconducting transition temperature $\left(T_{c}\right)$ is not affected significantly by $x$ up to $x$ $=0.05$ and latter decreases slightly for higher $x>0.05 . R(T)$ vs $H$ measurements show higher $T_{c}$ values under same applied magnetic fields for the nanodiamond added samples, resulting in higher estimated $H_{c 2}$ values. From the magnetization measurements, it was found that irreversibility field value $H_{\text {irr }}$ for the pristine sample is $7.5 \mathrm{~T}$ at $4 \mathrm{~K}$ and the same is increased to $13.5 \mathrm{~T}$ for $3 \mathrm{wt} \% \mathrm{nD}$ added sample at the same temperature. The $J_{c}(H)$ plots at all temperatures show that $J_{c}$ value is lowest at all applied fields for pristine $\mathrm{MgB}_{2}$ and the sample doped with $3 \mathrm{wt} \% \mathrm{nD}$ gives the best $J_{c}$ values at all fields. These results are discussed in terms of extrinsic pinning due to dispersed $n$-diamond in the host $\mathrm{MgB}_{2}$ matrix along with the intrinsic pinning due to possible substitution of $\mathrm{C}$ at boron site and increased interband scattering for highly doped samples resulting in extraordinary performance of the doped system. (C) 2008 American Institute of Physics.
\end{abstract}

[DOI: $10.1063 / 1.2831778]$

\section{INTRODUCTION}

The improvement of the pinning behavior in $\mathrm{MgB}_{2}$ is being considered as a key issue in the superconductivity field; many attempts have been made in this direction for enhancing the pinning strength of $\mathrm{MgB}_{2}$. In the last five years, various carbon sources, ${ }^{1-3}$ silicates, ${ }^{4}$ single elements, ${ }^{5,6}$ carbohydrates, ${ }^{7}$ organic acids, ${ }^{8}$ and other compounds ${ }^{9,10}$ have been doped in $\mathrm{MgB}_{2}$ and reported. It has become more clear that structural defects with dimensions at nanoscale play an important role for flux pinning, i.e., nanoparticle doping is the most effective approach to improve the superconducting performance of $\mathrm{MgB}_{2}$ and making $\mathrm{MgB}_{2}$ acceptable for practical applications. ${ }^{11}$ Nanoparticle doping can modify structure and electronic properties such as $T_{c}$, upper critical field and its anisotropy, gap widths, inter- and intraband scattering, defect structure, etc. ${ }^{12-14}$ In present paper, we are discussing the effect of nanodiamond addition on polycrystalline $\mathrm{MgB}_{2}$, i.e., how the superconducting performance of pristine sample is modified by addition of $n$-diamond.

\section{EXPERIMENT}

The $\mathrm{MgB}_{2}$-nanodiamond composites with composition $\mathrm{MgB}_{2}-n \mathrm{D}_{x}(x=0 \%, 1 \%, 3 \%, 5 \%, 7 \%$, and $10 \%)$ are synthesized by solid-state reaction route with ingredients of $\mathrm{Mg}, \mathrm{B}$, and $n$-diamond (see details in Ref. 15). The x-ray diffraction

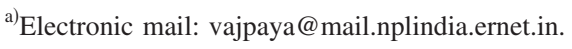

pattern of the compound was recorded with a diffractometer using $\mathrm{Cu} K \alpha$ radiation. The magnetoresistivity, $\rho(T) H$, was measured with $H$ applied perpendicular to current direction, using the four-probe technique on the Quantum Design physical property measurement system (PPMS). Magnetization measurements are carried out with a Quantum Design superconducting quantum interference device (SQUID) magnetometer MPMS-7.

\section{RESULTS AND DISCUSSIONS}

$\mathrm{X}$-ray diffraction patterns show that all the samples of $\mathrm{MgB}_{2}-n \mathrm{D}_{x}$ series are of nearly single phase with only a small quantity of unreacted $\mathrm{MgO}$ in them. For highest $n \mathrm{D}_{x}$ sample, i.e., $x=10 \mathrm{wt} \%$, the main intensity peak is broadened slightly (patterns not shown). The lattice parameters remain nearly invariant for all the samples until $x=5 \mathrm{wt} \%$ for $\mathrm{MgB}_{2}-n \mathrm{D}_{x}$. For 10 wt $\%$ sample, slight decrease in " $a$ " is seen. It means that, for this higher concentration, some diamond particles has got broken into carbon atoms, which substituted at boron site resulting in slight decrease in " $a$," but at low concentration, there is not such indication of breakage of $n$-diamond.

Variation of resistance with temperature under applied magnetic fields of up to $8 \mathrm{~T} R(T) H$ is shown in Fig. 1 (only transition zone). As we can see from this figure, with increasing $n$-diamond concentration, the superconducting onset temperature remains unchanged and the transition width changes slightly. This indicates that no substitution has taken place in 


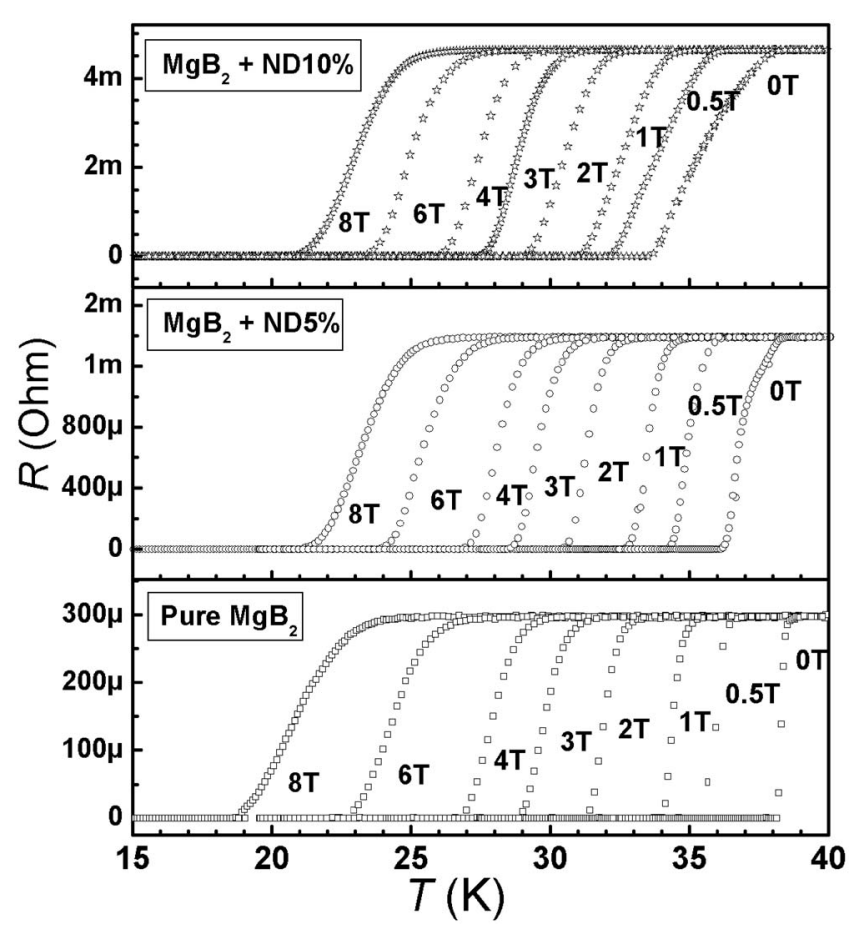

FIG. 1. Superconducting transition zone of resistance vs temperature plot under applied magnetic field $R(T) H$ up to $8 \mathrm{~T}$ for pure, $5 \mathrm{wt} \%$, and $10 \mathrm{wt} \%$ nanodiamond added samples.

this system. It is noted that $R(T) H$ curves for the doped samples shifted with increasing field much more slowly than the undoped one. For pure $\mathrm{MgB}_{2}$, the $T_{c}(R=0)$ value is observed at 38 and $18 \mathrm{~K}$ in 0 and $8 \mathrm{~T}$ applied fields, respectively. Interestingly, the $T_{c}(R=0)$ for $\mathrm{MgB}_{2}-5 \mathrm{wt} \% n \mathrm{D}$ sample in comparison to pure $\mathrm{MgB}_{2}$ is lowered to $36 \mathrm{~K}$ in zero applied field, the same is increased to $22 \mathrm{~K}$ under $8 \mathrm{~T}$ field.

Now, to see the effect of $n$-diamond addition on the upper critical field $H_{c 2}$, using resistivity variation under magnetic field plots, we estimated the $H_{c 2}$ values, i.e., $H_{c 2}$ obtained from the $90 \%$ values of the resistive transitions. The variation of $H_{c 2}$ with respect to temperature is not shown here (see Ref. 15), which demonstrates that, on increasing the concentration of $n$-diamond, a clear shift in upper direction can be achieved. The behavior of 3 and $5 \mathrm{wt} \%$ $n$-diamond added samples is quite competitive; but since the $T_{c}$ of $5 \% n \mathrm{D}$ is lower than $3 \% n \mathrm{D}$, we can say that $5 \% n \mathrm{D}$ sample shows better improvement in $H_{c 2}$ value. The formation of nanodomain structures is due to the presence of dispersed diamond in parent $\mathrm{MgB}_{2}$ grains (we have reported the high resolution transmission electron microscopy (HRTEM) and energy dispersive (ED) pattern in another paper ${ }^{15}$ ) causing the disorder in lattice. These nanodomains, which are comparable in size $(\sim 8-10 \mathrm{~nm})$ with the coherence length of $\mathrm{MgB}_{2}$ could result in strong in-plane and out-of-plane scatterings and contribute to the increase of $H_{c 2}$ value in a wide temperature regime.

In Fig. 2 the magnetic hysteresis loop for all the doped samples $(x=0 \%, 1 \%, 3 \%, 5 \%, 7 \%$, and $10 \%)$ at $10 \mathrm{~K}$ is shown in applied field up to $13 \mathrm{~T}$. This tells that the closing of hysteresis loop for pure $\mathrm{MgB}_{2}$ is at $\sim 6.5 \mathrm{~T}$, whereas the

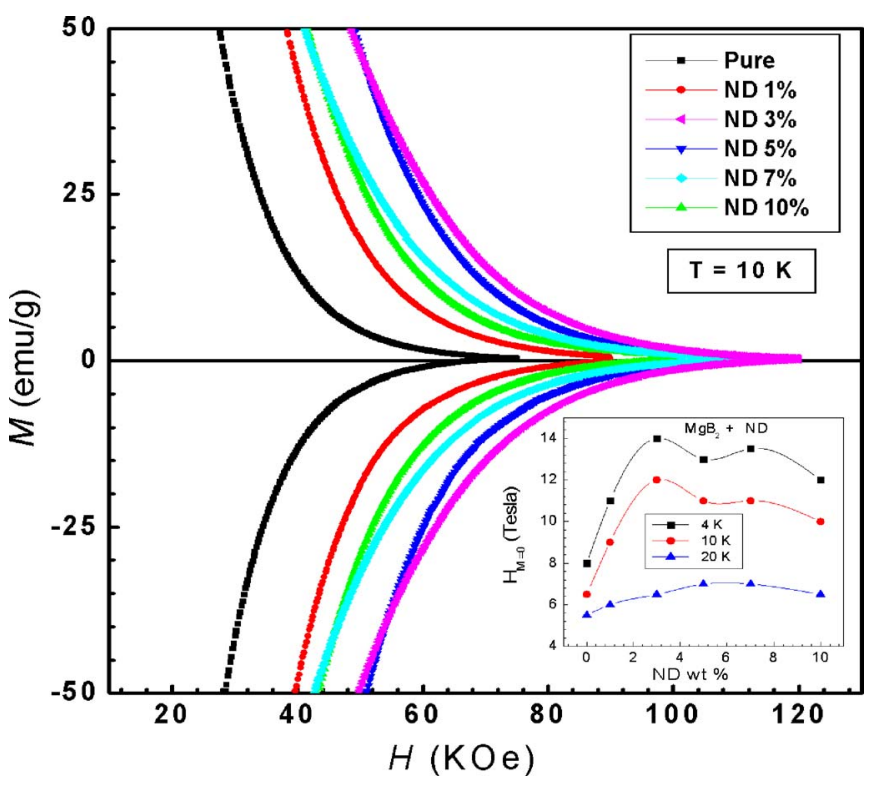

FIG. 2. (Color online) Magnetization loop $M(H)$ at $10 \mathrm{~K}$ for $\mathrm{MgB}_{2}-n \mathrm{D}_{x}$ $(x=0 \%, 1 \%, 3 \%, 5 \%, 7 \%$, and $10 \%)$ up to $12 \mathrm{~T}$ field, and inset shows the behavior of irreversibility field $H_{\text {irr }}$ with respect to $n$-diamond concentration at 4,10 , and $20 \mathrm{~K}$.

same is opened up to $\sim 11 \mathrm{~T}$ for $3 \mathrm{wt} \%$ doped sample. This indicates that there will be significant improvement in irreversibility field values for the doped samples. The irreversibility fields $\left(H_{\text {irr }}\right)$ is the one at which the magnetic hysteresis loop is nearly closed with $M \approx 0$. The $H_{\text {irr }}$ versus $x$ (concentration on nanodiamond) plot is shown in inset of Fig. 2 at 4, 10 , and $20 \mathrm{~K}$. The best values of $H_{\text {irr }}$ among these diamond added samples $\left(\mathrm{MgB}_{2}-n \mathrm{D}_{x}\right)$ is found for the sample with $x$ $=3 \%$. The $H_{\text {irr }}$ values for the undoped samples are 7.5, 6.5, and $4.5 \mathrm{~T}$ at 4,10 , and $20 \mathrm{~K}$, respectively; the same is increased up to $13.5,11.3$, and $5.5 \mathrm{~T}$ for the $3 \mathrm{wt} \% n$-diamond added sample, which reveals that nanodiamond doping significantly improved the $H_{\text {irr }}$ values. These results show that diamond doping enhances the flux pinning strength in parent $\mathrm{MgB}_{2}$ significantly. These results are in well agreement with previously reported data. ${ }^{16}$

Using $M(H)$ loop we estimated the critical current density by invoking Bean's critical state formula. Figure 3 shows the magnetic critical current density $\left(J_{c}\right)$ versus applied field $(H)$ at 20 and $10 \mathrm{~K}$. For all the samples (doped and undoped), $J_{c}$ attains the value greater than $10^{5} \mathrm{~A} / \mathrm{cm}^{2}$ at low fields at both the temperatures. At $20 \mathrm{~K}, J_{c}$ falls to $100 \mathrm{~A} / \mathrm{cm}^{2}$ at $4.5 \mathrm{~T}$ for pure $\mathrm{MgB}_{2}$, whereas for rest of the samples, this $J_{c}$ value is reached only at nearly $6 \mathrm{~T}$. At $20 \mathrm{~K}$, the $J_{c}$ is improved by an order of magnitude for doped samples as compared to the pure $\mathrm{MgB}_{2}$ at high fields, but for doped samples, all the plots are merging nearly at the same field $(6 \mathrm{~T})$, i.e., not much difference in $J_{c}(H)$ behavior is seen for doped samples at higher fields. For $10 \mathrm{~K}$, the situation is quite different at higher fields, i.e., the rate of $J_{c}$ drop is smaller than for all samples compared to undoped $\mathrm{MgB}_{2}$. The $J_{c}$ is 40 times higher than pure $\mathrm{MgB}_{2}$ at $10 \mathrm{~K}$ at $6 \mathrm{~T}$ applied field in case of $3 \mathrm{wt} \% n$-diamond added sample and $J_{c}$ value is still of the order of $10^{3} \mathrm{~A} / \mathrm{cm}^{2}$ at $10 \mathrm{~T}$ for the same sample. These results are quite comparable as reported 


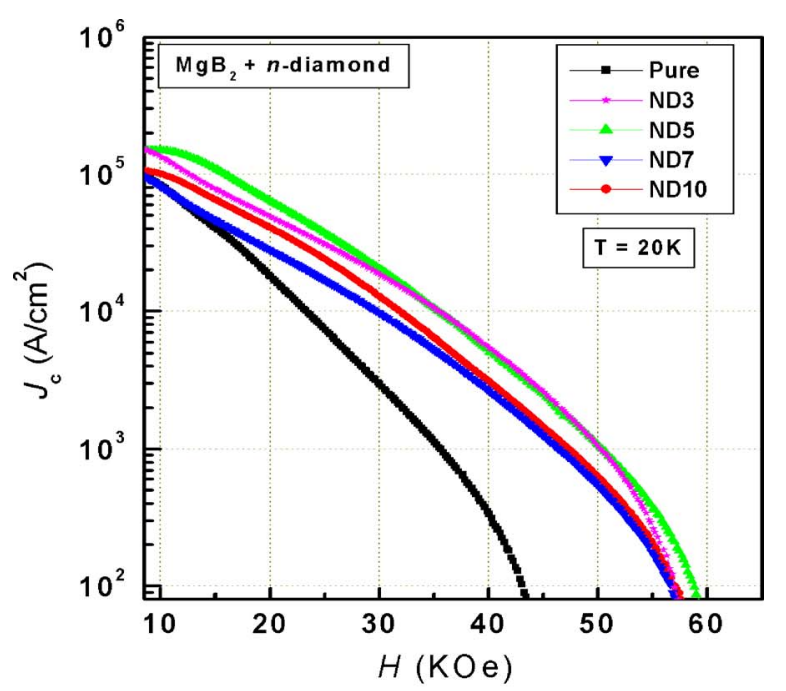

(a)

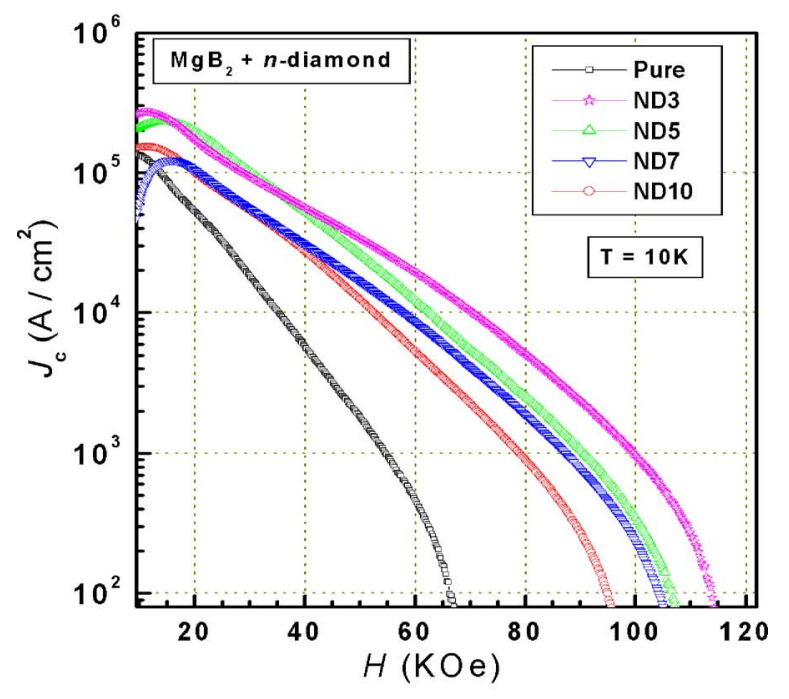

(b)

FIG. 3. (Color online) Critical current density $\left(J_{c}\right)$ variation with respect to applied magnetic field $(H)($ a) at $20 \mathrm{~K}$ and (b) at $10 \mathrm{~K}$.

earlier. ${ }^{17}$ The better $J_{c}(H)$ performance of $n$-diamond added samples indicates towards that the additional impurities at nanoscale introduced by $n$-diamond serve as strong pinning centers to improve flux pinning in higher applied fields.

To confirm the improved flux pinning behavior through diamond doping, the field dependence of normalized flux pinning force $\left(F_{p} / F_{p \text {, max }}\right)$ is shown in Fig. 4 at $10 \mathrm{~K}$. The relationship between flux pinning force and critical current density could be described by ${ }^{18}$

$$
F_{p}=\mu_{0} J_{c}(H) H,
$$

where $\mu_{0}$ is the magnetic permeability in vacuum. This figure depicts that the pinning forces are significantly improved than the pure $\mathrm{MgB}_{2}$ above $2 \mathrm{~T}$ for $n$-diamond doped samples because curves for the doped samples are shifted towards the higher fields, indicating enhanced flux pinning force in high fields. As revealed by the microstructure analysis, ${ }^{15}$ nanodiamond particles of size around $\sim 10 \mathrm{~nm}$ are dispersed in

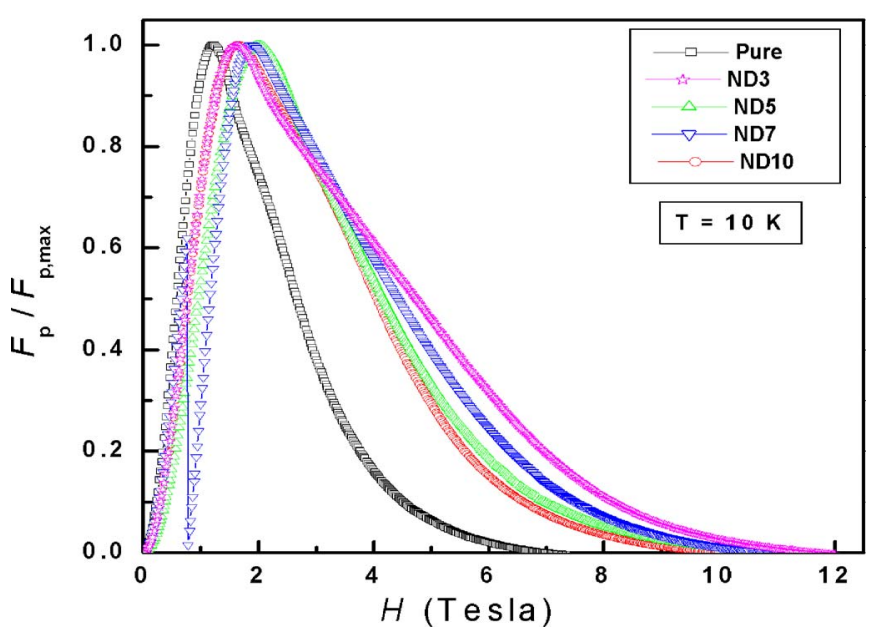

FIG. 4. (Color online) Variation of reduced flux pinning force $\left(F_{p} / F_{p \text {, max }}\right)$ with magnetic field for $\mathrm{MgB}_{2}-n \mathrm{D}_{x}(x=0 \%, 1 \%, 3 \%, 5 \%$, and $10 \%)$ at $10 \mathrm{~K}$.

$\mathrm{MgB}_{2}$ matrix. Because these particles have the size comparable to the coherence length of $\mathrm{MgB}_{2}$, it is highly possible that they may work as point pinning centers, causing a shift of the curve in $F_{p} / F_{p \text {,max }}$ vs $H$ curve towards the higher field. It can be seen from Fig. 4 that the peak corresponding to 3 wt $\% n$-diamond doped sample is much broader than those of other samples, indicating highest pinning strength in this sample at higher fields, which is consistent with the results of $J_{c}$ (Fig. 3).

In summary, the present work shows that nano-diamond doping is a promising way to fabricate high performance $\mathrm{MgB}_{2}$ bulk material with excellent values of $J_{c}(H), H_{c 2}$, and $H_{\text {irr }}(T)$.

\section{ACKNOWLEDGMENTS}

Dr. Rajeev Rawat from CSR-Indore is acknowledged for the resistivity under magnetic field measurements. Mr. Kranti Kumar and Dr. A. Banerjee are acknowledged for the high field magnetization measurements. Further, DST, Government of India is acknowledged for funding the 14 T PPMSVSM at CSR, Indore. The authors from NPL would like to thank Dr. Vikram Kumar (DNPL) for his constant encouragement.

${ }^{1}$ S. X. Dou et al., Appl. Phys. Lett. 83, 4996 (2003).

${ }^{2}$ R. H. T. Wilke et al., Phys. Rev. Lett. 92, 217003 (2004).

${ }^{3}$ S. X. Dou et al., Phys. Rev. Lett. 98, 097001 (2007).

${ }^{4}$ Y. Ma et al., Supercond. Sci. Technol. 16, 852 (2003).

${ }^{5}$ S. Y. Li et al., Physica C 363, 219 (2001).

${ }^{6}$ J. Y. Xiang et al., Physica C 386, 611 (2003).

${ }^{7}$ J. H. Kim et al., Appl. Phys. Lett. 89, 142505 (2006).

${ }^{8}$ J. H. Kim et al., Supercond. Sci. Technol. 20, 715 (2007).

${ }^{9}$ T. M. Shen et al., Supercond. Sci. Technol. 18, L49 (2005).

${ }^{10}$ A. Vajpayee et al., Physica C 466, 46 ( 2007).

${ }^{11}$ M. Nishiyama et al., Supercond. Sci. Technol. 17, 1406 (2003).

${ }^{12}$ C. Krutzler et al., Phys. Rev. B 74, 144511 (2006).

${ }^{13}$ M. Angst et al., Phys. Rev. B 71, 144512 (2005).

${ }^{14}$ S. Lee et al., Physica C 397, 7 (2003).

${ }^{15}$ A. Vajpayee et al., Supercond. Sci. Technol. 20, S155 (2007).

${ }^{16}$ C. H. Cheng et al., Supercond. Sci. Technol. 16, 1182 (2003).

${ }^{17}$ C. H. Cheng et al., Supercond. Sci. Technol. 20, 296 (2007).

${ }^{18}$ E. Martinez et al., Phys. Rev. B 75, 134515 (2007). 